\title{
A Reduced Electrically-Equivalent Model of the IEEE European Low Voltage Test Feeder
}

This paper was downloaded from TechRxiv (https://www.techrxiv.org).

\section{LICENSE}

CC BY 4.0

SUBMISSION DATE / POSTED DATE

$11-10-2021 / 14-10-2021$

CITATION

Khan, Maman Ahmad; Hayes, Barry (2021): A Reduced Electrically-Equivalent Model of the IEEE European Low Voltage Test Feeder. TechRxiv. Preprint. https://doi.org/10.36227/techrxiv.16785832.v1

$\mathrm{DOI}$

10.36227/techrxiv.16785832.v1 


\title{
A Reduced Electrically-Equivalent Model of the IEEE European Low Voltage Test Feeder
}

\author{
Maman Ahmad Khan, Graduate Student Member, IEEE, Barry Hayes, Senior Member, IEEE
}

\begin{abstract}
This letter presents a reduced, electricallyequivalent model of the IEEE European Low Voltage Test Feeder for use in distribution network studies. The original test feeder is made up of 906 buses, of which only 55 have loads connected. This work proposes an equivalent 116 bus network which accurately represents all of the characteristics of the original test feeder, but significantly reduces the computational effort required when applied in a range of distribution system applications. The model reduction technique applied is explained in detail, and the performance of the modified network is tested under a wide range of network loading conditions. The analysis in this letter demonstrates that the modified 116 bus network produces identical results with $80 \%$ less computation time when compared to the original 906 bus network.
\end{abstract}

Index Terms-Low voltage network, power distribution network, time-series power flow, topology reduction

\section{INTRODUCTION}

$\mathbf{T}$ HE analysis and planning of Low Voltage (LV) distribution networks have become important with the inclusion of smart meters at the consumer level and with the introduction of small consumer-based generation. The detailed understanding of the operating condition by the Distribution System Operators (DSOs) is important for the management and control of the distribution networks. This has created a requirement for researchers to develop and test LV network models for various applications. However, a major limitation for researchers at present is the availability of standard European LV test feeders. The only IEEE test feeder currently available for LV network studies is the IEEE European Low Voltage Test Feeder [1]. It is been widely used for low voltage studies since its introduction in 2015. This feeder is typically used for time series simulations that require a large computational effort. Therefore, efforts are being made to modify the standard IEEE European test feeder for reducing the modelling and computational efforts required for a number of applications.

The major limitation of previous related work in the literature is that it does not maintain the integrity (all the network properties) of the network. As can be seen in the reduction technique [2], loops are added in the network, which changes the radial configuration of the LV network. The work in [3] lacks the equivalent voltage values after the network reduction. The proposed technique in [4] uses assumptions like fixed current loads and the addition of synthetic load buses in the network. Some benchmark techniques like Minimum Spanning Tree (MST) reduction technique [5] work on retaining the three-phase lateral rather reducing the network. The reduction technique in [6] is computationally burdensome.

Accordingly, this letter presents an equivalent model that produces identical results with a greatly reduced modelling and computational effort. The modification of the standard IEEE 906 LV network is done without assuming any synthetic inclusion of line or load data configuration. The algorithm used to develop the modified network retains the standard IEEE 906 line and load data set properties. The power flow comparison has been shown between the standard IEEE 906 LV test feeder and the modified novel 116 bus feeder to compare the network performance. The data set of the modified 116 bus network is provided with the paper [7].

\section{Methodology}

This section describes the framework and techniques used to develop the reduced equivalent IEEE LV European network. Initiating from the standard grid model which introduces the topological elements, the reduction approach and the clustering of the reduces topology has been elaborated in detail.

\section{A. Grid Modeling}

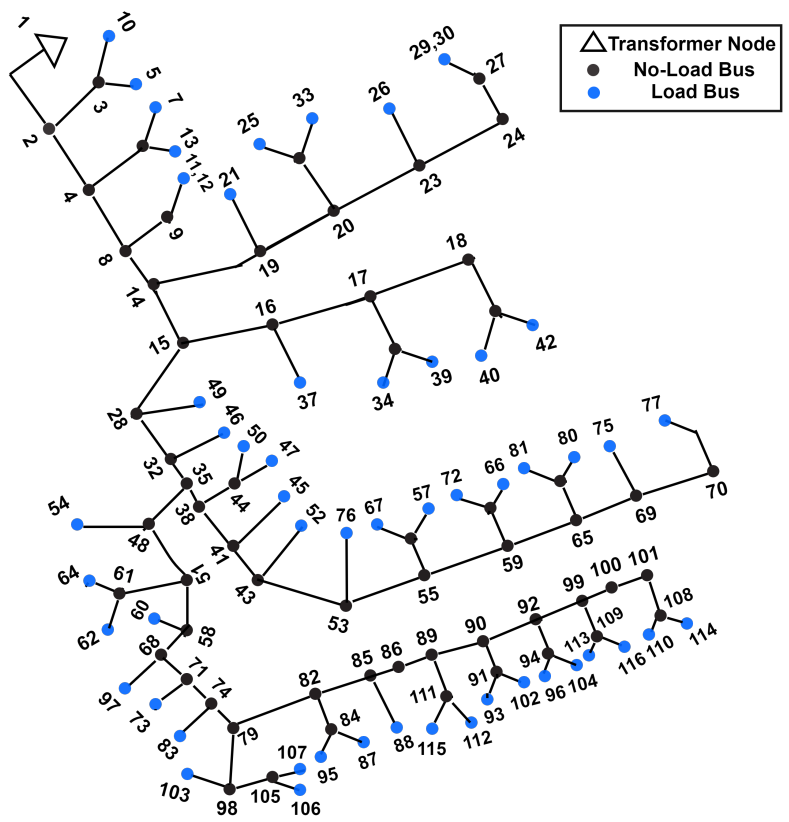

Fig. 1: Modified IEEE-906 LV Test feeder

The IEEE LV European test feeder consists of 906 low voltage nodes, connected by 905 branches along with 55 load buses [1]. The given LV network test bench is available with the time series data for 55 single phase loads, 21 for the phase A, 19 for the Phase B, 15 for the phase C. This LV feeder is provided with 416 phase-to-phase voltage level with a base 
frequency of $50 \mathrm{~Hz}$. Distribution lines are defined by line codes which are specified by sequence impedances and admittances. The loads are taken as constant PQ loads with base loads specified with 1 kilowatt $(\mathrm{kW})$ and 0.95 Power Factor $(\mathrm{PF})$ at peak loading condition. These loads are defined with the load shapes of 24 hour data with 1-minute time interval for time-series simulation.

As seen from the Table I pseudocode, a reduced equivalent test feeder has been modelled by proposing a reduction technique in which all the no-load buses on the branch consisting of same line data having the same line configuration are added together on the same branch of the feeder. In all branches of the network, the no-load buses are aggregated to reduce the size of the network. The load data set information remains intact along with the no-load nodes where the branches split into the sub-branches reaching the load buses, as shown in Fig. 1. This implies that the modified network aggregates all the lines from the substation to the end terminals, which consists of load buses. The load data, load shape, and load location have been kept the same as that of the standard IEEE 906 network with no assumption of reducing or modifying the load in the model structure. The dataset of the modified 116 Bus network has been provided with this paper on IEEE DataPort [7]

TABLE I: Equivalent Reduced Model Pseudocode

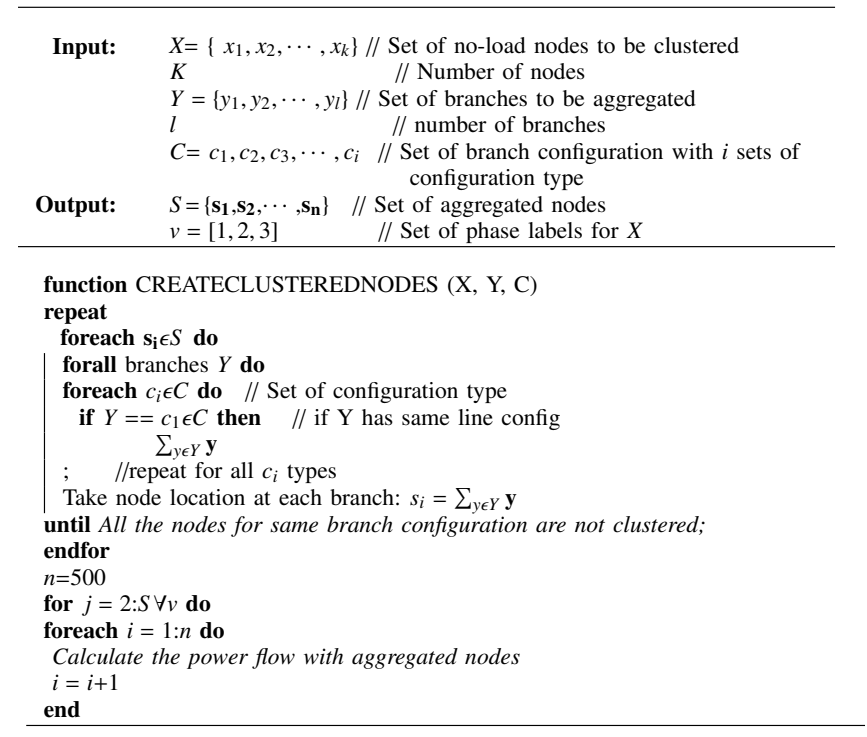

\section{B. Performance Evaluation}

To check the validity of the modified network, the timeseries power flow algorithm has been simulated on both the networks, the standard IEEE 906 network and the modified 116 bus network, with the help of an open source EPRI software OpenDSS. The load data consists of 1-minute time interval dataset for 24 hours. To ensure that the modified network is an equivalent model for the standard IEEE 906 network under realistic conditions, the time-series power flow simulation is run for different loading conditions- at high loading condition with given IEEE 906 LV feeder load dataset; medium loading; and low loading conditions. The COM interface is used to perform power flow at different loading condition using OpenDSS engine. This algorithm is run for 500 times for each loading condition in MATLAB and the mean computational time is obtained. The Table II below shows the time-series power flow output comparison between the two networks at peak loading condition.

TABLE II: Time-series power flow output comparison

\begin{tabular}{ccc}
\hline Circuit Summary & $\begin{array}{c}\text { IEEE 906 Bus } \\
\text { Network }\end{array}$ & $\begin{array}{c}\text { Modified 116 } \\
\text { Bus Network }\end{array}$ \\
\hline Buses & 907 & 117 \\
Loads & 55 & 55 \\
Nodes & 2721 & 351 \\
Mean Computational & 7.88 & 1.64 \\
Time & 3 & 3 \\
Total Iterations & 1.0499 & 1.0499 \\
Max Pu. Voltage & 1.045 & 1.045 \\
Min Pu. Voltage & $1.05441 \times 10^{-2}$ & $1.05441 \times 10^{-2}$ \\
Total Active Power (MW) \\
Total Reactive Power \\
(Mvar) & $3.4673 \times 10^{-3}$ & $3.4673 \times 10^{-3}$ \\
Total Active Losses & $2.84957 \times 10^{-5}$ & $2.84957 \times 10^{-5}$ \\
(MW) & & $1.09987 \times 10^{-5}$ \\
Total Reactive Losses \\
(Mvar)
\end{tabular}

The summary of the time-series power flow performed for these two different feeders proves that the modified 116 bus network is an equivalent reduced version of the standard IEEE $906 \mathrm{LV}$ test feeder. The unbalanced $\mathrm{AC}$ power flow is run 500 times. As it can be seen from Table [II the modified 116 is $80 \%$ faster in running power flow as compared to the standard IEEE 906 test feeder. The mean computation time for simulating power flow at different loading conditions for both the given networks using $2.5 \mathrm{GHz}$ processor with $12 \mathrm{~GB}$ RAM is shown in Table III

TABLE III: Mean Computational Time

\begin{tabular}{ccc}
\hline Loading Condition & $\begin{array}{c}\text { IEEE-906 Bus } \\
\text { Network }\end{array}$ & $\begin{array}{c}\text { Modified 116 } \\
\text { Bus Network }\end{array}$ \\
\hline $\begin{array}{c}\text { Low Loading } \\
(25 \%)\end{array}$ & $6.98 \mathrm{sec}$ & $1.518 \mathrm{sec}$ \\
$\begin{array}{c}\text { Medium Loading } \\
(50 \%)\end{array}$ & $7.32 \mathrm{sec}$ & $1.58 \mathrm{sec}$ \\
$\begin{array}{c}\text { High Loading } \\
(100 \%)\end{array}$ & $7.88 \mathrm{sec}$ & $1.64 \mathrm{sec}$ \\
\hline
\end{tabular}

\section{Proposed Reduced Network Applications}

The proposed reduced network can be used to perform Low Voltage State Estimation (LVSE). The original standard IEEE 906 European network cannot be used for this application due to the network's lack of sufficient measurement information. With a more accurate real-time network model obtained through distribution system state estimation, other operational functions such as active and reactive power optimisation, network restoration, load balancing, and optimal network configuration can be more reliably tested [8]. The proposed reduced network significantly reduces the amount of 

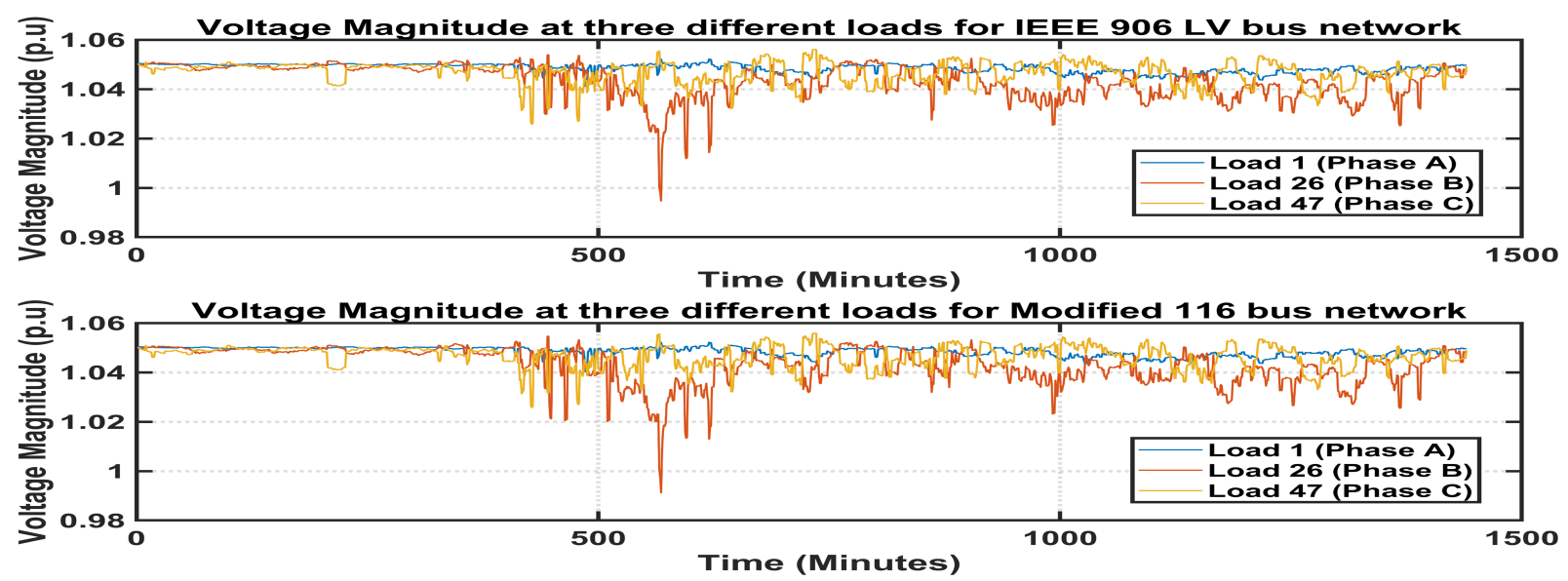

Fig. 2: Voltage magnitude for each of the three phases at Load 1 (Phase A), Load 26 (Phase B) and Load 47 (Phase C)

data to be exchanged and the processing time required locally compared to the original IEEE LV network [4]. As shown in the previous section, the 24-hour, 1-minute interval time-series load flow is run 500 times for different loading conditions, and the reduced network is $80 \%$ faster than the original IEEE 906 LV network. Therefore, the reduced network can be used for applications that require different case studies to run like QSTS (Quasi-Static Time-Series) for DER integration and hosting capacity studies. This network is also much more efficient for applications requiring Monte-Carlo analysis, such as network reliability studies. A range of other monitoring and control applications can be tested on this reduced network with a much-reduced computational burden.

The proposed network can also be used to study local electricity markets more efficiently since the reduced model will reduce the average delivery path length (by reducing the number of no-load buses) when transferring energy from provider to consumer; also the study of energy losses of delivery can be studied more efficiently with respect to the original IEEE 906 LV network [9]. Other applications such as distributed energy pricing can be studied on this proposed reduced network which may not be feasible on the original network due to the large number of nodes and complexity of the network [4].

\section{Results}

The time series power flow algorithm is run in MATLAB using COM interface with OpenDSS engine as mentioned in the previous section. The voltage magnitude at three different load buses for three phases are shown in the Fig. 2 placed at three different locations.

Phase A for load 1 is at bus 5 in modified 116 network and at bus 34 in IEEE 906 network, where phase B for load 26 is at bus 60 in the modified network and at bus 522 in the standard IEEE 906 network, and the last, phase C for load 47 is at bus 104 in the modified 116 network and at bus 822 for IEEE 906 bus network, which is at the far end from the substation.

The absolute error for the three phases at three different load buses shows the difference between the IEEE 906 power flow voltage magnitude results and the modified 906 network power flow results. As it can be seen from the Fig. 3 the maximum absolute error between the two defined network is within the specified limit for the time series power flow.

The voltage under different loading conditions when run in MATLAB for 500 times are plotted as boxplots in Fig. 4 for three different phases.

The load data for peak loading condition is taken as $1 \mathrm{~kW}$ with $0.95 \mathrm{PF}$ and $0.13 \mathrm{~kW}$ with same PF for low loading condition. As it can be seen from the figure, all the three phases tend to have high voltages under low demand conditions with wide spread of voltages during high demand conditions. The power flow output voltages do not violate the European standard limit of 0.94 - 1.1 p.u for LV networks.
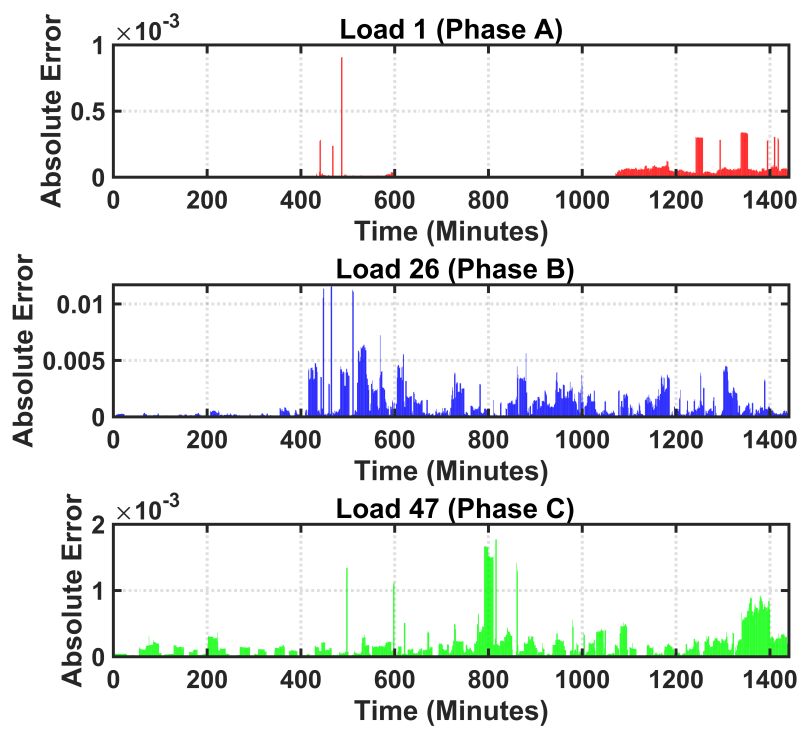

Fig. 3: Absolute error difference between IEEE 906 and Modified 116 voltage magnitude 

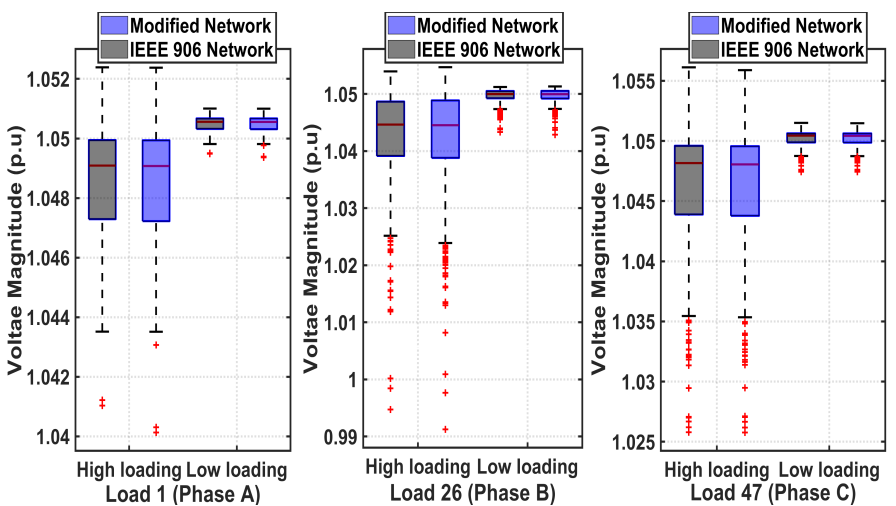

Fig. 4: Box plots for three phases at different loading conditions

\section{Conclusion}

This letter presents a modified IEEE European LV network with reduced topology, suitable for a range of LV network modelling and analysis applications. The numerical power flow results demonstrate an electrically equivalent, reduced model of the original IEEE $906 \mathrm{LV}$ network, which mitigates against the limitations of the original network in various distribution network applications. It also reduces computation time by up to $80 \%$ compared to using the original network, while providing identical power flow results. Furthermore, this modified network is shown to be robust for all loading conditions. The full data set for the modified network is provided in [7].

\section{REFERENCES}

[1] IEEE, "Distribution test feeders," IEEE PES Distribution Systems Analysis Subcommittee Radial Test Feeders, May, 2015.

[2] A. Prostejovsky, O. Gehrke, M. Marinelli, and M. Uslar, "Reduction of topological connectivity information in electric power grids," in $201651 \mathrm{st}$ International Universities Power Engineering Conference (UPEC), 2016, pp. $1-6$.

[3] H. Oh, "Aggregation of buses for a network reduction," IEEE Transactions on Power Systems, vol. 27, no. 2, pp. 705-712, 2012.

[4] Z. K. Pecenak, H. V. Haghi, C. Li, M. J. Reno, V. R. Disfani, and J. Kleissl, "Aggregation of voltage-controlled devices during distribution network reduction," IEEE Transactions on Smart Grid, vol. 12, no. 1, pp. $33-42,2020$.

[5] A. Nagarajan and R. Ayyanar, "Application of Minimum Spanning Tree algorithm for network reduction of distribution systems," 2014 North American Power Symposium, NAPS 2014, 2014.

[6] A. Nagarajan, A. Nelson, K. Prabakar, A. Hoke, M. Asano, R. Ueda, and S. Nepal, "Network reduction algorithm for developing distribution feeders for real-time simulators," IEEE Power and Energy Society General Meeting, pp. 1-5, January,2018.

[7] M. Khan and B. Hayes, "For paper - a reduced electrically-equivalent model of the ieee european low voltage test feeder," 2020. [Online]. Available: https://dx.doi.org/10.21227/0d2n-j565

[8] M. Huang, Z. Wei, M. Pau, F. Ponci, and G. Sun, "Interval state estimation for low-voltage distribution systems based on smart meter data," IEEE Transactions on Instrumentation and Measurement, vol. 68, no. 9, pp. 3090-3099, 2018.

[9] M. R. Alam, M. St-Hilaire, and T. Kunz, "Peer-to-peer energy trading among smart homes," Applied energy, vol. 238, pp. 1434-1443, 2019. 